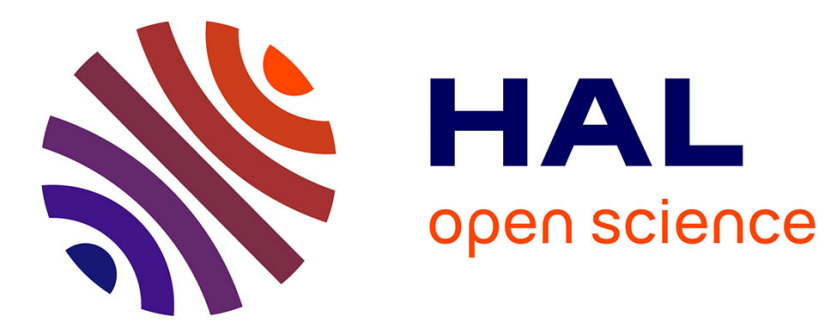

\title{
D'un foncier consumé à une réintégration des terres agricoles
}

\author{
Guillaume Schmitt, Nicolas Rouget, Magalie Franchomme
}

\section{To cite this version:}

Guillaume Schmitt, Nicolas Rouget, Magalie Franchomme. D'un foncier consumé à une réintégration des terres agricoles. Études rurales, 2018, 201, pp.166 - 191. 10.4000/etudesrurales.12377 . halshs03163310

\section{HAL Id: halshs-03163310 \\ https://shs.hal.science/halshs-03163310}

Submitted on 9 Mar 2021

HAL is a multi-disciplinary open access archive for the deposit and dissemination of scientific research documents, whether they are published or not. The documents may come from teaching and research institutions in France or abroad, or from public or private research centers.
L'archive ouverte pluridisciplinaire HAL, est destinée au dépôt et à la diffusion de documents scientifiques de niveau recherche, publiés ou non, émanant des établissements d'enseignement et de recherche français ou étrangers, des laboratoires publics ou privés. 


\section{études Études rurales \\ rurales $201 \mid 2018$ \\ Sur les terrains du foncier}

\section{D’un foncier consumé à une réintégration des terres agricoles}

Le cas de la métropole lilloise

From consumed land to the reintegration of farmland: The case of metropolitan

Lille

\section{Guillaume Schmitt, Nicolas Rouget et Magalie Franchomme}

\section{CpenEdition}

Journals

Édition électronique

URL : http://journals.openedition.org/etudesrurales/12377

DOI : 10.4000/etudesrurales. 12377

ISSN : $1777-537 \mathrm{X}$

Éditeur

Éditions de l'EHESS

Édition imprimée

Date de publication : 1 janvier 2018

Pagination : 166-191

ISBN : 978-2-7132-2748-6

\section{Référence électronique}

Guillaume Schmitt, Nicolas Rouget et Magalie Franchomme, « D'un foncier consumé à une

réintégration des terres agricoles », Études rurales [En ligne], 201 | 2018, mis en ligne le 01 janvier

2020, consulté le 06 janvier 2021. URL : http://journals.openedition.org/etudesrurales/12377 ; DOI :

https://doi.org/10.4000/etudesrurales.12377

(C) Tous droits réservés 


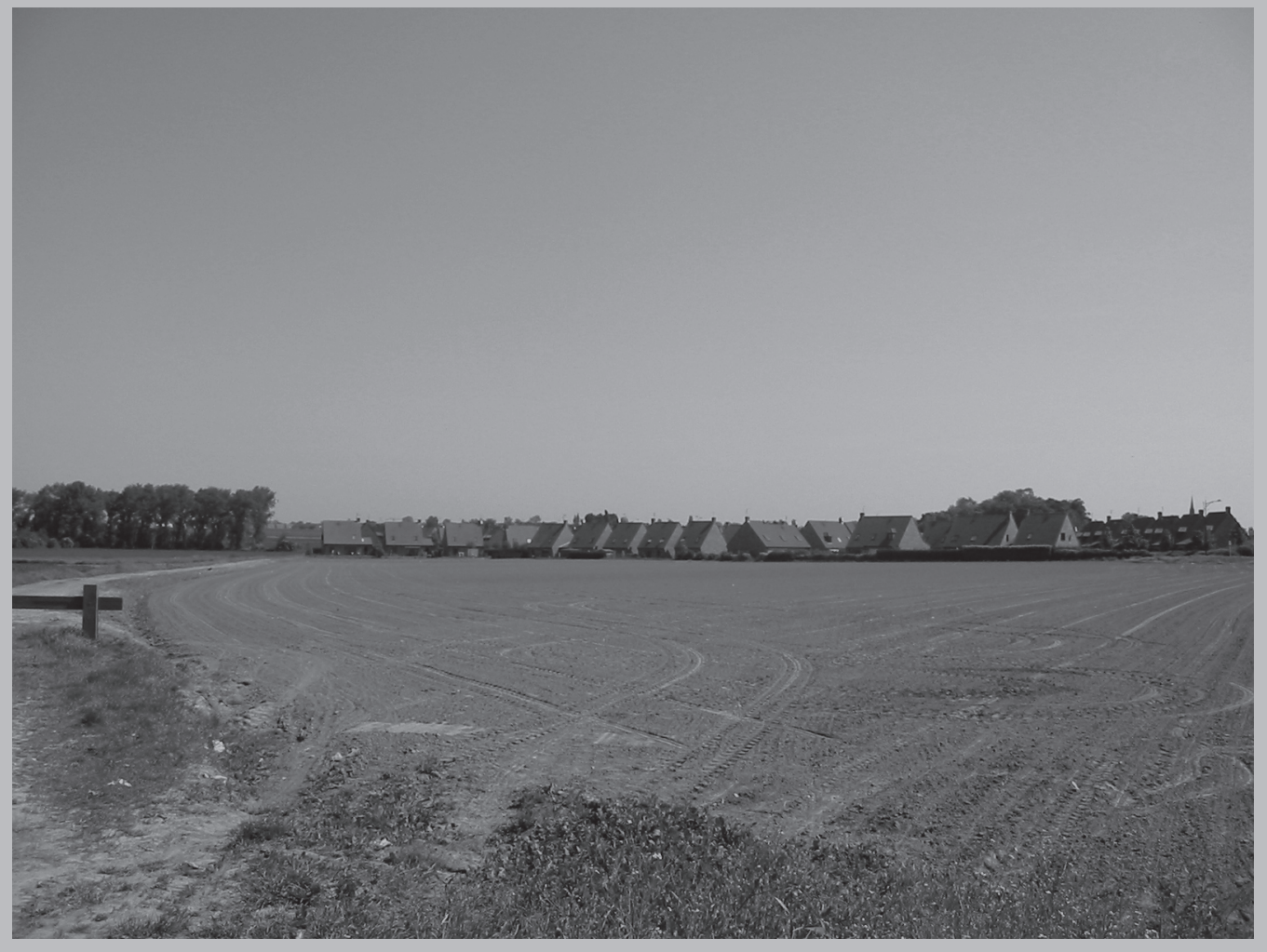

Commune de Templemars, département du Nord (mai 20I3). Photo: N. Rouget. 


\section{D'un foncier consumé}

\section{à une réintégration des terres agricoles}

\section{Le cas de la métropole lilloise}

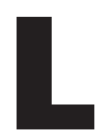

'accroissement des demandes sociales, portant tant sur l'offre de «nature» que sur des questions de sécurité alimentaire, redéfinit les relations ville-campagne. Ces reconfigurations renforcent la réintégration des terres agricoles au «métabolisme urbain» [Barles 2002] dans ses dimensions territoriales et politiques. Cette intégration s'opère néanmoins, pour la métropole de Lille comme pour les autres en France, dans un contexte de périurbanisation, d'élargissement des compétences des établissements publics de coopération intercommunale (EPCI), de promotion et de mise en concurrence des métropoles.

Avec un objectif de to ooo hectares voués à la nature et aux loisirs à l'horizon $2015^{\mathrm{I}}$, le schéma directeur (SD) de 2002 de la métropole lilloise est en soi une évolution dans le modèle de planification territoriale. Là, où le précédent schéma limitait le rôle des espaces naturels à celui d'infrastructures vertes (contribution à la régulation des crues des zones humides...), celui de 2002 s'applique à davantage réglementer leurs usages, à limiter les possibilités de conversion et projette d'étendre leur superficie par des processus de restauration écologique. Cette ambition est une réponse à la demande grandissante des populations citadines ${ }^{2}$ de pouvoir jouir d'espaces verts à proximité de leur domicile [Boutefeu 2005]. Les coupures urbaines constituent également un argument reconnu et non négligeable de promotion de la qualité du cadre de vie des grandes métropoles pour accueillir des grandes firmes mettant en concurrence les territoires à l'échelle mondiale [Harvey I989].

I. Objectif récemment reporté à 2020 .

2. Voir L. Bourdeau-Lepage et R. Vidal, «Nature urbaine en débat: à quelle demande sociale répond la nature en ville?», Le Déméter, 2013, p. 195-210. 
À l'automne 2002, Lille métropole ${ }^{3}$ est la première à se doter en France d'une compétence «espace naturel métropolitain, valorisation du paysage» 4 . Le manque d'espaces verts publics, environ $\mathrm{I}_{5} \mathrm{~m}^{2}$ par habitant dans les années I990, explique ce choix. Récemment, la loi du 27 janvier 2014 (dite «loi Mapam») a renforcé cette compétence et introduit notamment de nouvelles responsabilités en matière de gestion des milieux aquatiques.

Par «espaces naturels», il convient d'entendre des espaces ouverts définis comme un ensemble d'espaces agricoles et d'espaces verts selon des approches post-fonctionnaliste et néonaturaliste cherchant à étendre les fonctions récréatives des friches aux terres agricoles situées en périphérie d'agglomération [Lévy 2010; Margetic et al. 20I6]. Ainsi près d'un tiers des espaces identifiés par la politique «espaces naturels» sont des terres agricoles où, durant la première décennie du $\mathrm{xxI}^{\mathrm{e}}$ siècle, a été expérimentée une série de mesures pour concilier production agricole, périurbanisation, valorisation des paysages, tourisme de proximité et protection de la ressource en eau. Enfin, les récents travaux du schéma de cohérence territoriale $(\mathrm{Scot})^{5}$ mettent en lumière le retour à une production alimentaire de proximité et l'importance de l'agriculture dans l'activité économique.

Ces évolutions ne semblent pas, toutefois, spécifiques à la métropole lilloise et sont plébiscitées dans de nombreuses agglomérations [Aubry 20I3] et le retour à une agriculture de proximité en est l'un des principaux arguments. La mobilisation de l'idée de production alimentaire de proximité semble être un moyen de (re)lier l'agriculture et les consommateurs au territoire, tout en faisant émerger un consensus et une stratégie collective alliant acteurs économiques, représentants de la profession, édiles et usagers [Torre 20I4]. Néanmoins, une série de facteurs confèrent à la métropole lilloise un intérêt singulier: réflexion ancienne sur le renouvellement des liens ville-campagne, diversité des acteurs et des expérimentations (parcs périurbains, programme paysager, «sacralisation» d'espaces agricoles, lotissement maraîcher et horticole...) et caractéristiques de l'espace envisagé (urbanisation et industrialisation précoces, imbrication des espaces) ou des modes d'organisation locaux ${ }^{6}$.

Aussi, devons-nous voir dans les trajectoires des politiques foncières de la métropole lilloise une volonté de plus en plus affirmée de ménager

\footnotetext{
3. Lille métropole communauté urbaine (LMCU), qui compte 85 communes, est devenue au titre de la loi de modernisation de l'action publique territoriale et d'affirmation des métropoles du 27 janvier 20I4 (dite «Mapam») Métropole européenne de Lille (MEL) le Ir janvier 20I5. Elle compte aujourd'hui 90 communes.

4. Délibération du conseil communautaire du 20 novembre 2000.

5. L'arrêt du projet a été signé par le Comité syndical du Scot de Lille Métropole le 26 février 2016.

6. Voir B. Bonduelle, Lettre aux 86 maires de Lille, Lille, Éditions La Voix du Nord, I992, p. 245 .
} 
des espaces naturels et agricoles désormais englobés dans un vaste espace métropolitain? Ces politiques consacrent-elles un retour à l'essentiel (protéger la terre nourricière) ou, au contraire, traduisent-elles une complexification du débat où l'alimentation serait le point de convergence des aspirations héritées et des préoccupations contemporaines?

Le présent article propose de revenir dans un premier temps sur le traitement des terres agricoles dans la métropole lilloise depuis I950 pour illustrer la logique dominante du gisement foncier et la subordination des marchés fonciers ruraux et périurbains aux processus de «résidentialisation». Les phases et trajectoires agricoles significatives des recompositions territoriales à l'œuvre, déterminées à partir de l'analyse des schémas d'aménagement depuis i97I et des dynamiques de filière, sont exposées dans un deuxième temps. Enfin, à partir de l'exemple d'un projet d'aménagement (parc de la Deûle), nous observons que l'articulation entre ville, agriculture et nature est non seulement en cours de reconfiguration, mais renouvelle également le cadre de travail des acteurs autour de l'enjeu foncier (les édiles passant, dans les orientations stratégiques, d'une nature jardinée à une agriculture nourricière de proximité).

\section{Le foncier agricole: support privilégié de développement de la périurbanisation}

Saisir l'enjeu foncier dans l'agglomération lilloise demande une réflexion multiscalaire, puisqu'il est traité par une superposition d'échelons administratifs, allant de l'échelle infra-communale à la région urbaine Lille Nord Pas-de-Calais ${ }^{7}$, en passant par les périmètres de l'intercommunalité, de l'arrondissement, de la métropole transfrontalière et de l'aire métropolitaine [Paris 2002]. Le périmètre de l'actuel schéma de cohérence territorial correspond à celui de l'arrondissement et incorpore également des communes alentour. Le diagnostic territorial du Scot intègre les différentes échelles spatiales des dynamiques foncières ${ }^{8}$. Pour autant, l'adjectif qualificatif «foncier», érigé en nom par dérivation lexicale, n'est pas aisé à qualifier. Étienne le Roy le définit comme:

7. Cette dernière s'étend du littoral à l'ouest aux communes belges densément peuplées au nord jusqu'à la limite du département du Nord à l'est.

8. À titre d'illustration, 53 références «au foncier» sont recensées dans Scot Lille Métropole. Le diagnostic et le débat du comité syndical du 22 novembre 2010 (contre 47 dans le schéma directeur de 2002), dont voici quelques extraits: «pour renforcer son attractivité pour les entreprises, l'arrondissement doit pouvoir proposer une offre immobilière et foncière répartie de façon équilibrée sur le territoire» (p. 65), «protection du foncier agricole» (p. 69) ou «Nous avons ici un foncier qui n'existe pas ailleurs, à condition de le rendre disponible. Il me semble que c'est un facteur formidable d'attractivité» (p. I62). 
l'ensemble particulier de rapports sociaux ayant pour support la terre ou l'espace territorial. Ces rapports sociaux sont principalement déterminés par les facteurs économiques (accumulation primitive de capital et extraction de rente), juridiques (normes d'appropriation et modalités de règlement des conflits) puis par les techniques d'aménagement pouvant matérialiser et caractériser ces rapports sociaux en autant de régimes distincts. [I99I: I3-I4].

Pour suivre l'évolution des différentes facettes du foncier agricole au cours du temps dans la métropole lilloise, six sources de données ont ainsi été consultées. Des cartographies de l'utilisation du sol réalisées par interprétation de photographies aériennes et d'images satellitales (I990, I998, 2005 et 2009) produites par le conseil régional Nord-Pas de Calais ${ }^{9}$ ont permis d'analyser les dynamiques territoriales. Nous avons également utilisé le zonage des documents d'urbanisme ${ }^{\text {I0 }}$ en I993, 2000, 2006 et 2016 sur I24 des $\mathrm{I} 34$ communes du périmètre du Scot de la métropole lilloise ${ }^{\mathrm{II}}$. Du fait des changements législatifs ${ }^{12}$ et des modalités locales de détermination des zonages réglementaires, nous avons regroupé les 224 types de zonage de notre secteur d'étude en quatre catégories ${ }^{\mathrm{I} 3}$ : urbain, à urbaniser, agricole et naturel. L'évolution des zonages illustre les changements juridiques et techniques dans le champ de l'aménagement du territoire. Par ailleurs, nous avons analysé les déclarations d'intentions d'aliéner (DIA) transmises à la Société d'aménagement foncier et d'établissement rural (Safer) Flandres-Artois dans le cadre de l'exercice de son droit de préemption sur

9. La qualité des données de référence étant sensiblement différentes, les cartographies ont été homogénéisées par généralisation de la sémantique [Comber et al. 2004; Devillers et Jeansoulin 2005]. La résolution sémantique a été simplifiée en trois catégories (zones urbaine, agricole et naturelle) en s'appuyant sur la nomenclature emboitée définie dans le programme de cartographie harmonisée à l'échelle européenne Corine Land Cover [Bousquet et al. 2013]. La dernière cartographie en vigueur est en 52 catégories d'occupation/utilisation du sol (<http://www.sigale.nordpasdecalais.fr/DICO/D_Ocsfinog.html>).

Io. Plan d'occupation des sols (POS) et plan local d'urbanisme (PLU).

II. Les données n'étaient en effet pas disponibles à toutes les dates sur l'ensemble de la zone d'étude. Les documents d'urbanisme actuels sont en cours de numérisation au format SIG (Système d'information géographique) dans la région des Hauts-de-France aux standards CNIG (Conseil national de l'information géographique). Cette numérisation a été amorcée avant l'ordonnance du 20 décembre 2013 relative à l'amélioration des conditions d'accès aux documents d'urbanisme et aux Servitudes d'utilité publique (SUP). Cette ordonnance crée le géoportail national de l'urbanisme et oblige les auteurs de documents d'urbanisme et de SUP à alimenter ce dernier par des versions électroniques (art. Li29-I, 2 et 3 du Code de l'urbanisme). 12. Par exemple, citons le passage du POS au PLU initié par la loi Solidarité et renouvellement urbains du I3 décembre 2000. Schématiquement, les POS sont généralement présentés comme des documents dont l'objectif général est l'organisation de l'urbanisation, tandis que les PLU seraient plutôt des documents contraignants davantage cette dernière [Leroux 20IO]. I3. Nous avons repris la norme de regroupement proposée par le CNIG. 
les fonds agricoles ou les terrains à vocation agricole ${ }^{\mathrm{I} 4}$ sur la période 2002$2012^{15}$. Ces données sur les marchés fonciers sont révélatrices des facteurs économiques et des dynamiques territoriales. Les documents graphiques et littérales du cadastre de la direction générale des finances publiques (DGFIP) pour l'année 20I4, associés au plan parcellaire, nous ont permis d'aborder les facteurs juridiques et plus précisément les normes d'appropriation du foncier [Geniaux et Napoléone 20II]. Enfin, notre corpus a été complété par des statistiques du recensement général agricole (RGA) à différentes dates, par des enquêtes auprès d'exploitants agricoles menées depuis le début des années 2000 et par des entretiens semi-directifs ${ }^{16}$ conduits en 2016 avec des agents de la métropole lilloise, responsables des services «espaces naturels métropolitains », «agricultures et dynamiques territoriales» et «stratégie et opérations foncières ».

\section{Un gisement foncier, réserve de la croissance urbaine}

Le foncier agricole de la métropole lilloise apparaît singulier à double titre au regard de l'analyse des documents d'urbanisme qui se sont succédé depuis I990 et de l'évolution de l'utilisation du sol comparativement au reste de l'ancien Nord-Pas-de-Calais et des autres grandes métropoles françaises (voir fig. 1). Tout d'abord, le foncier agricole est nettement en retrait par rapport à la situation régionale. Il couvre $54,6 \%$ de la métropole lilloise contre environ $78 \%$ du Nord-Pas de Calais, mais surtout il se distingue par sa forte régression, six fois plus importante que la tendance régionale depuis les années $1990^{17}$. Bien que ces écarts se retrouvent dans d'autres secteurs très urbanisés où les activités et les services se concentrent dans les zones métropolitaines [Halbert 20Io], ils montrent la dynamique des mutations foncières. Elle s'illustre également dans les différents POS, puisque dans ceux de I993, plus de $65 \mathrm{~km}^{2}$ essentiellement occupés par des exploitations agricoles étaient ouverts à l'urbanisation. Ce potentiel de construction a atteint $72 \mathrm{~km}^{2}$ dans les POS en 2000 .

\footnotetext{
I4. Art. L-I43-I du Code rural et de la pêche maritime. I5. Voir J. Lachaud, SAFER: Les sociétés d'aménagement foncier et d'établissement Rural. Définition, Missions, Moyens d'action, Paris, France Agricole («Droits de l'entreprise agricole»), 2006. I6. Les entretiens (un par service) portaient sur six items: panorama des orientations et des actions emblématiques en termes d'agriculture; évolution des compétences de la métropole lilloise en termes d'espaces naturels et agricoles; organisation des services et direction; actions emblématiques sur les communes du parc de la Deûle; évolution récente de l'organigramme des services (voir infra); évolution des politiques portant sur les espaces naturels et agricoles suite à la réorganisation des services.

I7. Par exemple $-4 \%$ durant les années I990 contre - 0,6\% en moyenne dans les deux départements. Voir Le faire-part foncier de l'EPF Nord-Pas de Calais à la communauté urbaine de Lille, Établissement public foncier-Nord-Pas-de-Calais, 2007.
} 
Face à ce constat et du fait de la loi SRU, le SD de 2002 introduit une règle relativement simple avec deux tiers des constructions dans le tissu urbain existant et un tiers en extension. Les zones ouvertes à l'urbanisation sont ainsi quasiment divisées par deux dans les PLU de 2006. Cette règle est d'ailleurs confortée par le programme local de l'habitat (PLH de 2005 et 2012), pour lequel les zones ouvrant droit à la construction sont, en

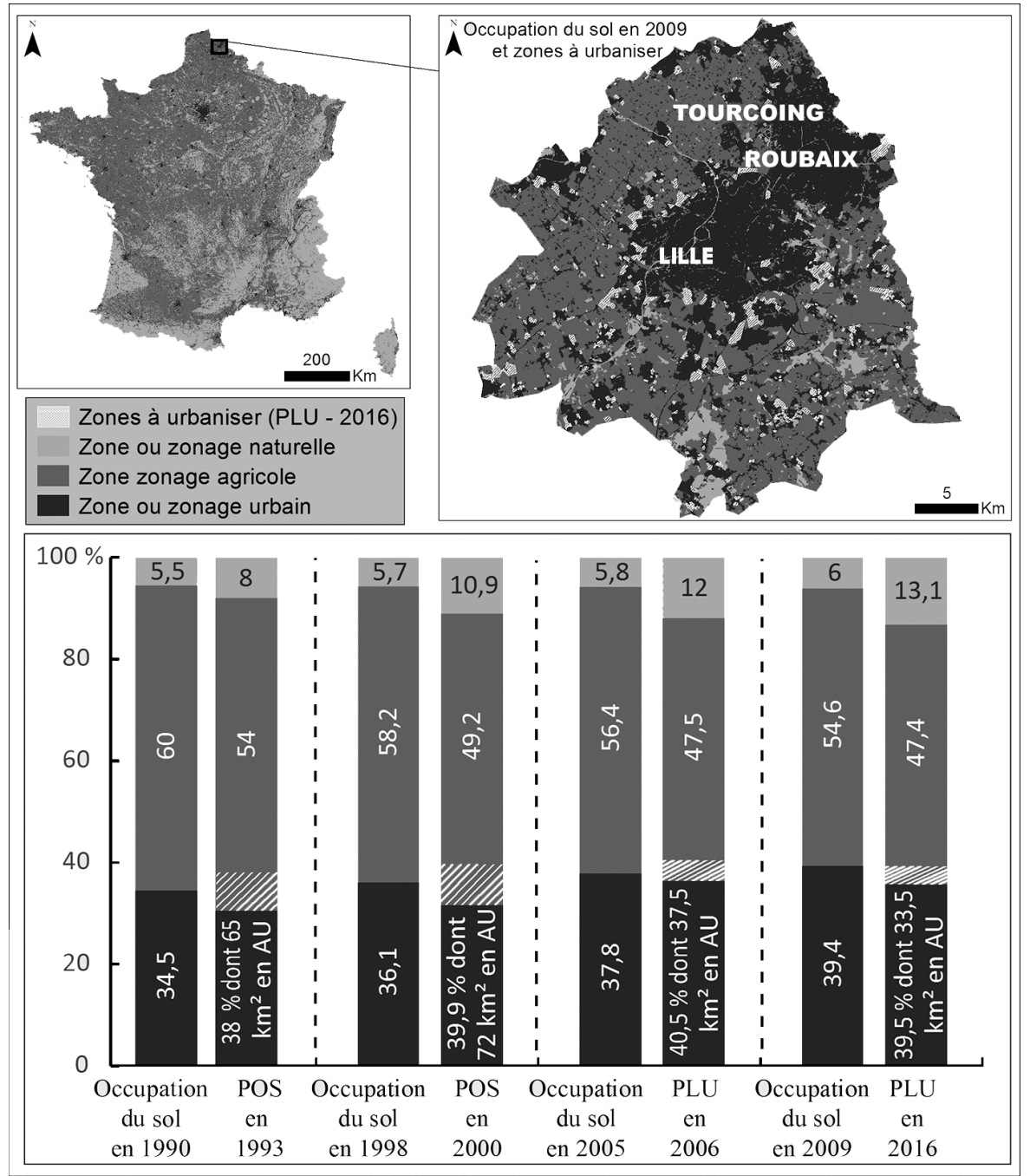

Figure 1. Évolution de l'utilisation du sol et des zonages des documents d'urbanisme dans la métropole lilloise depuis 1990. Réalisation: G. Schmitt. Sources: AEE, Corine and Cover, 2006; Métropole européenne de Lille (1993, 2000, 2006 et 2016); conseil régional des Hauts-de-France (1990, 1998, 2005 et 2009). 
$206^{18}$, réduites à $33,5 \mathrm{~km}^{2}$. Aussi, en comparaison avec d'autres métropoles, comme Lyon et Nantes où les surfaces agricoles couvrent approximativement $40 \%$ de leur territoire, celle de Lille atteint $54,6 \%$. Cette différence s'explique notamment par d'importantes constructions à des fins résidentielles aux dépens des terres agricoles dans les années I990 et 2000 dès que l'on s'éloigne de 15 ou 20 kilomètres du tissu urbain central. À Bordeaux, Nice, Marseille et Toulouse, de tels changements s'opèrent davantage dans un rayon de moins de to kilomètres [Schmitt 2009]. Larmature urbaine polycentrique du nord de la France concourt ainsi à diffuser et à diluer, dans un vaste ensemble territorial, les changements d'affectations foncières générées par l'attractivité métropolitaine [Paris I993].

Les écarts avec les autres agglomérations françaises tiennent aussi à l'urbanisation et à l'industrialisation précoces de la métropole lilloise et, en conséquence, à la faible proportion des espaces naturels [Franchomme 2008]. Ces derniers représentent $6 \%$ de l'espace métropolitain ${ }^{19}$. Par leur rareté et les enjeux en termes de promotion des territoires, et de réponse à la demande sociale de nature, les espaces naturels constituent des objets centraux de planification territoriale et sont donc souvent inscrits dans des zonages d'inventaire ${ }^{20}$, de protection ${ }^{2 \mathrm{I}}$ et dans des projets de requalification du cadre de vie à l'instar du parc de la Deûle au sud de Lille. L'évolution du zonage naturel dans les POS et les PLU depuis les années I990 est particulièrement significative (passant de $8 \%$ en I993 à I3,I\% en 20I6). Ainsi dans le SD de 2002, le constat de la faible présence d'espaces verts par rapport aux autres métropoles européennes $\left(\mathrm{I} 5 \mathrm{~m}^{2}\right.$ par habitant, contre $50 \mathrm{~m}^{2}$ à Amsterdam et $28 \mathrm{~m}^{2}$ à Bruxelles) est expliqué par une «inertie opérationnelle [...] dans les années 1970 et I980, des politiques de mise en valeur des espaces naturels ${ }^{22}$. En 20IO, le ratio par habitant passe à $23 \mathrm{~m}^{2}$ et tient au fait que «plusieurs centaines d'hectares d'espaces naturels et récréatifs ont été aménagées ou réhabilités en une dizaine d'années, transformant petit à petit l'image de la métropole» ${ }^{23}$. Ce chiffre reste cependant en deçà de la

I8. Signalons qu'entre le PLU de 2006 et celui de 2016 , plus de 500 révisions ont été effectuées. Avec le passage du schéma directeur au Scot, les lois Grenelle 2 du I2 juillet 20 Io et Alur (24 octobre 20I4), les PLU communaux sont en révision générale et une démarche de consultation publique préalable est en cours pour élaborer un PLU intercommunal (du I3 février 20I5 au I ${ }^{e r}$ avril 20I7). Le PLU intercommunal devrait être adopté en fin d'année 2019. 19. Selon l'inventaire réalisé par l'Agence d'urbanisme et de développement de Lille, la proportion des espaces naturels de la métropole lilloise est de $15 \%$. La différence s'explique principalement par l'utilisation d'autres sources de données, d'une méthode de photointerprétation différente et de l'inclusion des squares, des promenades vertes ou de bases de loisirs dans la catégorie «espace naturel».

20. Zones naturelles d'intérêt écologique faunistique et floristique (ZNIEFF).

2I. Espace boisé classé (EBC).

22. Voir le Schéma directeur de développement et d'urbanisme de Lille Métropole, 2002 (p. 92).

23. Voir Lille Métropole. Le diagnostic et le débat du comité syndical du 22 novembre 2010, p. I25. 
moyenne des 50 plus grandes agglomérations françaises qui est de $31 \mathrm{~m}^{2} /$ habitant d'après l'Union nationale des entreprises du paysage (l'Unep). Le diagnostic du Scot précise que la fragmentation des espaces naturels s'est accentuée et que ces derniers sont en général de faibles emprises ${ }^{24}$.

Le foncier agricole est le principal gisement de la construction dans la métropole lilloise, comme l'illustre l'urbanisation de la commune de Wavrin au sud-ouest de Lille (voir fig. 2). Cette urbanisation à l'échelle locale, à plus de $15 \mathrm{~km}$ du centre de Lille, s'explique par la réduction progressive des zonages «à urbaniser» à proximité immédiate de la ville et par les coûts techniques et financiers des transactions foncières en zone urbaine dense [Schmitt 2009].

\section{Des marchés fonciers agricoles sous constante influence urbaine}

La relation entre la rente foncière et la proximité urbaine n'est plus à démontrer [Guigou I982]. Les marchés fonciers et immobiliers de l'habitat et de la construction sont d'ailleurs étudiés, dans la nouvelle économie urbaine, comme des facteurs d'intervention directe sur les marchés fonciers agricoles $^{25}$ [Geniaux et Napoléone 2005]. La segmentation des marchés fonciers reste délicate, du fait de la faible disponibilité de données fiables et de présupposés quant à la destination des biens après leur mise en vente [Granelle 2009]. Cinq marchés fonciers relatifs à des parcelles agricoles sont généralement recensés ${ }^{26}$ : celui des terres restant agricoles (libres ou louées); des parcelles boisées, des landes et des étangs; des terres à vocation résidentielle (parcelles libres) et de loisirs (équitation...) ; de la «maison à la campagne» (anciennes fermes) et celui pour la construction (zones d'activités, de logements et infrastructures de transport). Selon cette typologie, les segments de marché à des fins non agricoles comptent un plus grand nombre de transactions, pour des montants financiers plus conséquents et des surfaces équivalentes dans la métropole lilloise (voir fig. 3).

Le marché des parcelles acquises à des fins résidentielles (terrain à aménager avant équipement et viabilisation) ou de loisirs est, en volume financier, dans la métropole lilloise cinq fois plus important que le marché de la terre agricole. Il représente, en revanche, des enjeux financiers moindres à l'échelle régionale et nationale. La proximité urbaine influence ainsi les dynamiques foncières agricoles de trois façons. Tout d'abord, elle concourt

\footnotetext{
24. La plus grande forêt de la métropole lilloise couvre 650 ha.

25. Mise en place d'indicateurs plus ou moins solides (voir les travaux de la FN Safer) ou détection des effets d'anticipation par exemple.

26. Voir Y. Gérard et G. Schmitt, «Bouge de là. L'observation en mouvement», Études foncières, $2012, n^{\circ}{ }^{156}$, p. 38-44.
} 

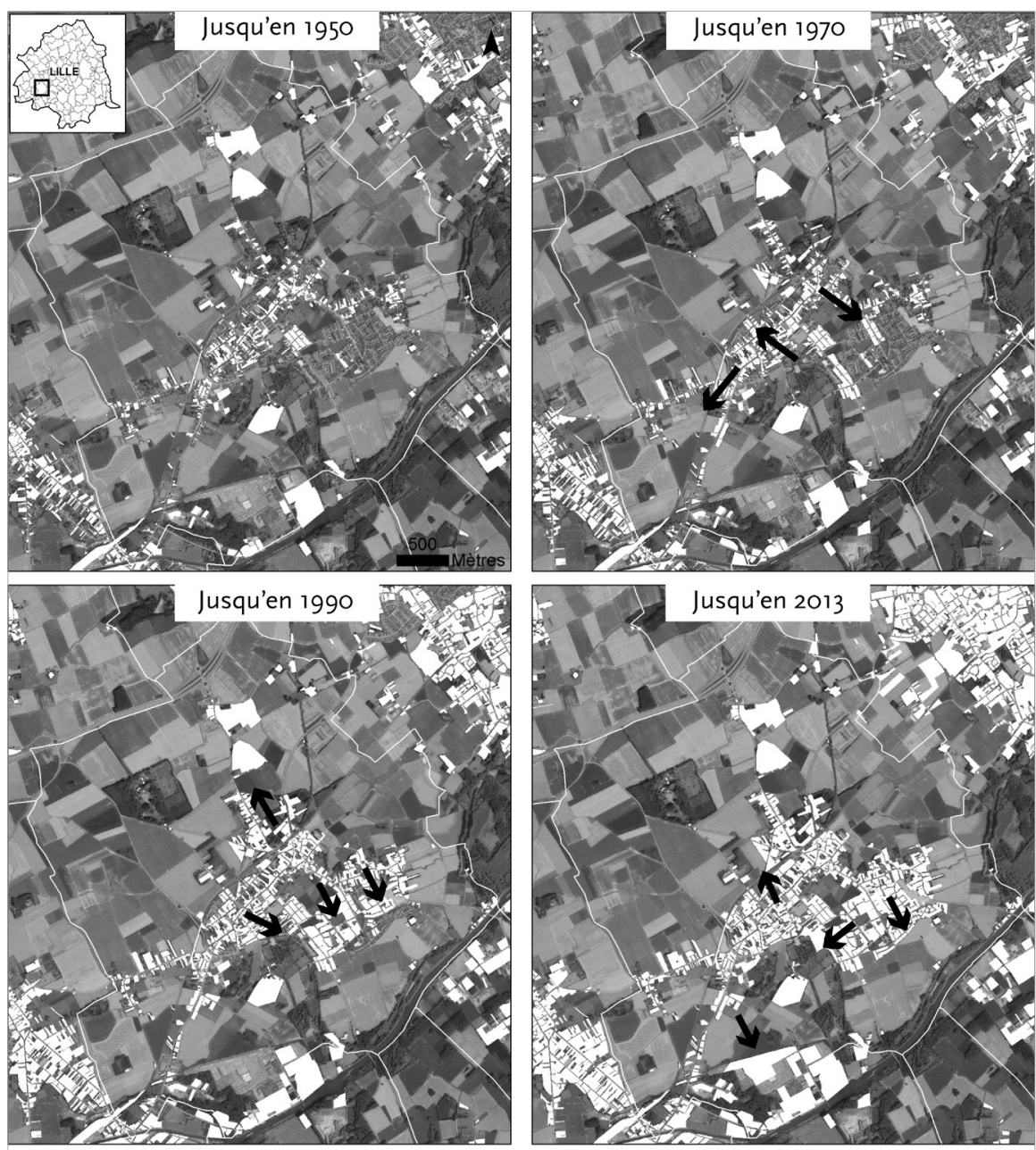

Parcelle support de construction d'habitations ou de locaux

Orientation privilégiée des constructions

Figure 2: Illustration de l'expansion spatiale du bâti dans la commune de Wavrin depuis I950. Réalisation: G. Schmitt. Sources: IGN Géofla (2016); orthophotographies conseil général du Nord (2013), DGFIP (2016). 


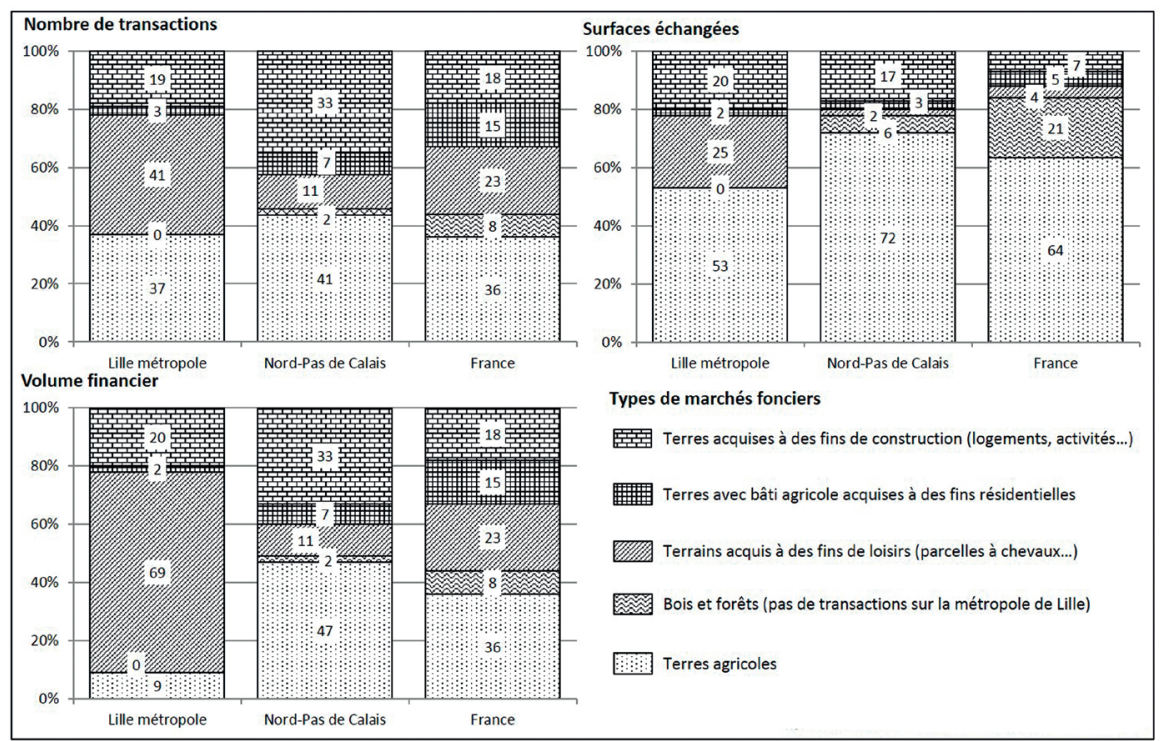

Figure 3: Les marchés fonciers des fonds et des terres à vocation agricole dans la métropole lilloise: des transactions pour l'urbanisation et les loisirs.

Réalisation: G. Schmitt. Sources: Safer Flandres-Artois (moyenne 2005-2012).

par perméabilité des marchés ${ }^{27}$ à maintenir des prix de transactions élevés pour les parcelles supports de productions agricoles. Selon l'enquête sur la valeur vénale des terres, l'hectare est, depuis une quinzaine d'années, plus de $50 \%$ plus cher dans le Nord-Pas de Calais que dans le reste de la France ${ }^{28}$ et près de $75 \%$ supérieur dans le secteur de Lille-Pévèle (entre l'agglomération et la frontière franco-belge). Ensuite, les terres agricoles et notamment les près et les prairies sont régulièrement acquises à des fins de loisirs ${ }^{29}$ par des personnes ne travaillant pas dans l'agriculture. Enfin les contraintes induites (franchissements routiers, cloisonnement, fragmentation et enclavement des parcelles cadastrales) contribuent à accentuer le morcellement d'un parcellaire agricole dont l'émiettement est particulièrement fort (voir fig. 4). Le marché des terres agricoles, dans un contexte de faire-valoir indirect très dominant, est par conséquent relativement peu ouvert avec, en proportion, moins de transactions et une tendance à l'agrandissement des exploitations moins prononcée.

27. Lors de la formulation de prix de référence par exemple ou la valeur d'opinion que constitue le prix de la terre.

28. Rappelons que cette enquête à dire d'experts repose sur des critères agronomiques, le rendement du fermage, les marges par hectare, la distance à la ville la plus proche...

29. En moyenne, quatre déclarations d'intention d'aliéner sur dix concernent le pâturage de chevaux, la pêche... 


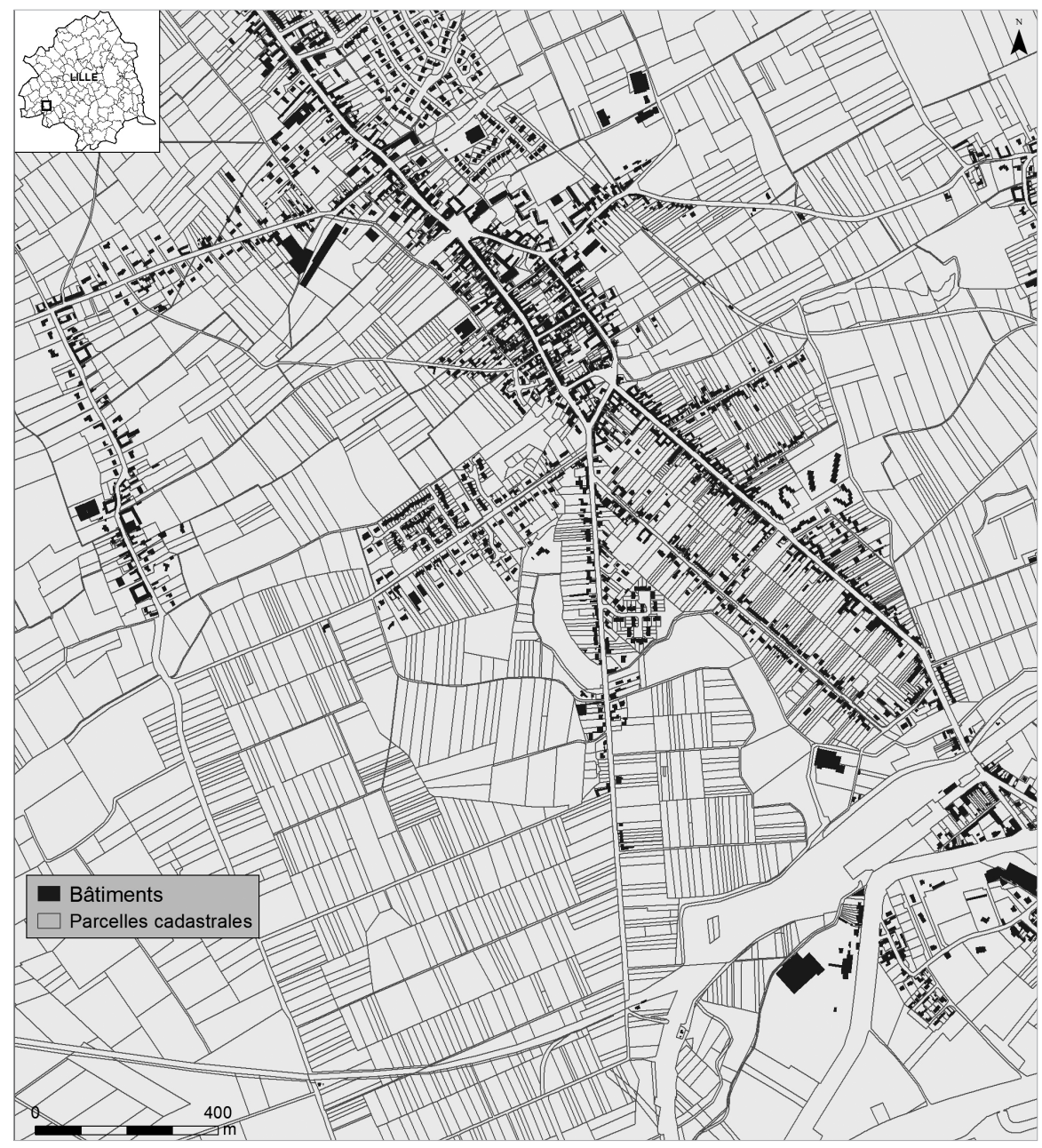

Figure 4. Un parcellaire agricole émietté et fragmenté (cadastre, Sainghin-en-Weppes, périphérie sud de Lille). Réalisation: G. Schmitt. Source: DGFIP (2016).

Les enquêtes conduites dans le Sud-Est lillois [Rouget 20I3] soulignent ainsi la faible part des stratégies agricoles d'adaptation et de résistance par l'agrandissement (moins d'une exploitation sur cinq). La SAU moyenne des exploitations de la métropole était, en 20I0, de 34,5 ha contre 6I ha à l'échelon des départements du Nord et du Pas-de-Calais ${ }^{30}$. Ce trait caractéristique de l'agriculture de la Métropole européenne de Lille (MEL) est à imputer également à une série de facteurs plus spécifiquement locaux. Parmi ces paramètres, figurent l'héritage endivier (qui a longtemps permis le maintien

30. D’après le diagnostic agricole de Lille Métropole de 20II. 
de structures foncières étroites) mais également la présence d'agriculteurs belges qui louent à l'année des surfaces agricoles parfois importantes ${ }^{31}$. Le processus de concentration des entreprises agricoles de la MEL en est d'autant plus ralenti qu'en raison du haut niveau de rémunération de ces locations, des agriculteurs - en fin de carrière ou double-actifs - procèdent à une rétention des terres ainsi mises à disposition.

\section{Des aspirations aménagistes et des trajectoires agricoles qui se redessinent}

Le foncier agricole est régi par des enjeux contradictoires puisqu'il est, d'une part, le support d'ambitions aménagistes et, de l'autre, un capital économique des exploitations agricoles. Cependant, ce contexte est en train de changer avec les courants de pensée dominants de l'aménagement du territoire et des perspectives renouvelées de l'agriculture (re)territorialisée.

\section{L'influence de la pensée aménagiste sur le foncier agricole}

Les formes multiples de périurbanisation sont sources de reconfigurations des considérations portées sur le foncier agricole [Martin et al. 2006]. De récents travaux ont permis de préciser l'évolution des paradigmes associés à la question foncière agricole dans les débats contemporains [Margetic et al. 20I6]. Sont ainsi repérables: un postulat agraire reposant sur des critères agronomiques centré sur les propriétaires exploitants, une entrée agroindustrielle où le foncier est support de production d'agents économiques, une approche néonaturaliste fondée sur des principes de préservation et de conservation (plus ou moins partagés par les acteurs de l'aménagement et de l'urbanisme) et un regard post-matérialiste où le foncier agricole est perçu comme l'expression d'intérêts individuels de production et de consommation contribuant au bien commun.

Nous proposons de reprendre cette grille de lecture en l'appliquant aux schémas d'aménagement qui se sont succédé depuis une quarantaine d'années dans la métropole lilloise (fig. 5). Cette reconfiguration s'appuie sur la lecture du schéma directeur d'aménagement et d'urbanisme (SDAU) de I971 ${ }^{32}$, du SD de $2002^{33}$ et des travaux du Scot approuvé en 20I7. Ces documents constituent des points cruciaux de la place du foncier agricole dans la pensée aménagiste. En effet, ils sont le produit d'une accumulation pendant

3I. $9 \%$ des SAU communales de I4 communes de l'Est lillois [Rouget 2008].

32. Voir, le Schéma directeur d'aménagement et d'urbanisme de l'arrondissement de Lille. Rapport justificatif. Agence d'urbanisme de la métropole du Nord, I97I.

33. Voir le Schéma directeur de développement et d'urbanisme de Lille Métropole de 2002 ainsi que sa version de 1997 . 


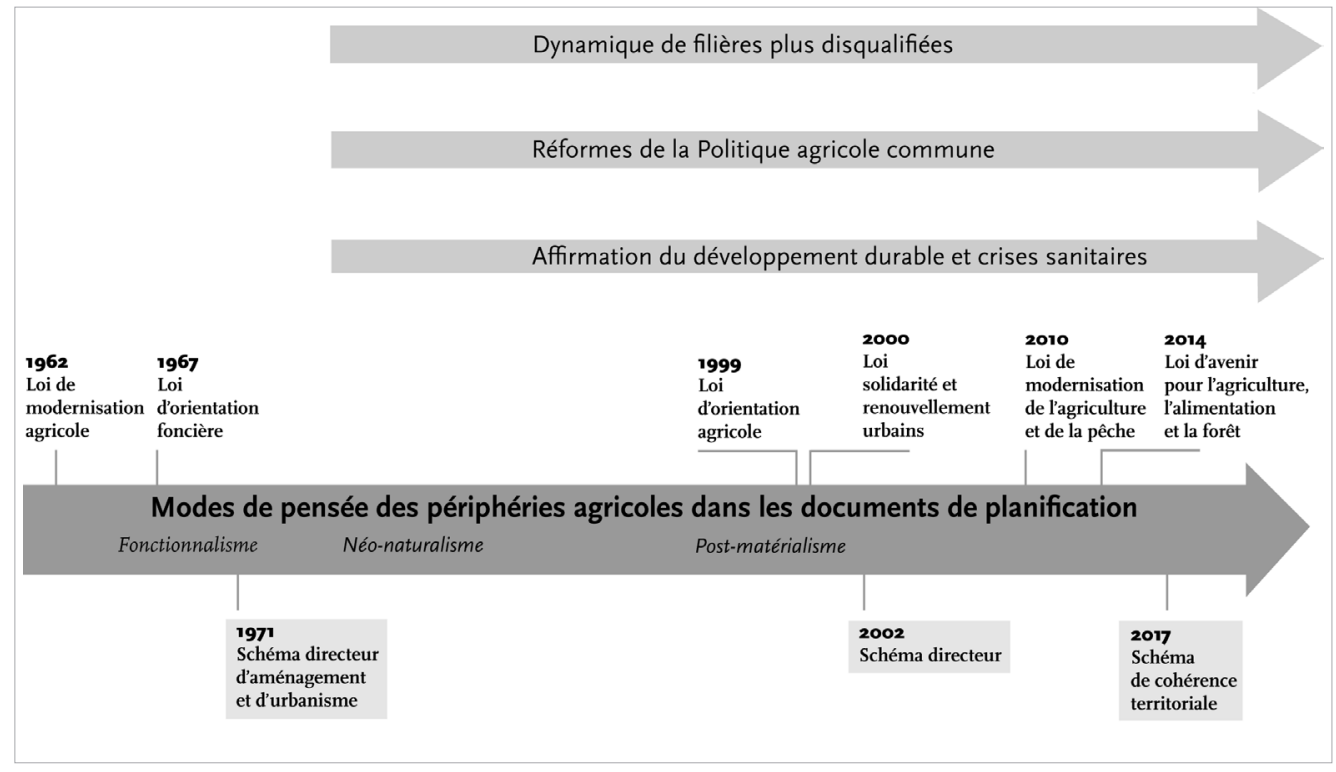

Figure 5: Les trois temps des modes de pensée du foncier agricole de la métropole lilloise.

les quarante dernières années, de connaissances et de projections de valeurs économiques, sociales et environnementales portées sur les terres agricoles. Les travaux d'Isabelle Estienne [2008] sur le paysage dans la métropole lilloise illustrent la filiation entre les différents documents et des travaux de prospective antérieurs à l'instar de ceux de l'Oream Nord ${ }^{34}$.

Ainsi les trois documents montrent trois inflexions dans les modes de pensée dominants quant au foncier agricole de la métropole lilloise. Le mode fonctionnaliste prend tout son essor avec le schéma hydraulique et d'emprise des zones d'équipement du projet de parc de la Deûle de l'Oream en I97635. Les terres agricoles bordières des équipements de gestion des eaux sont indiquées comme pouvant être protégées, afin d'assurer l'épuration des eaux par lagunage. L'approche néo-naturaliste a apporté une dimension plus environnementale au système de valeurs et adopté l'idée d'immuabilité quant à la mobilisation du foncier dans des projets de territoire. Ainsi dans le SD de $2002^{36}$, il est précisé que:

34. Aménagement d'une région urbaine: le Nord-Pas-de-Calais, Organisation d'études d'aménagement de l'aire métropolitaine (Oream) du Nord, I97I.

35. Le parc de la Deûle, note de présentation, Oream-Nord, 1976.

36. Schéma directeur de développement et d'urbanisme de Lille Métropole, 2002, Syndicat mixte Lille métropole, p. I9I. 
les sites naturels d'intérêt régional et métropolitain représentent une richesse écologique, faunistique et floristique mais aussi un intérêt esthétique, récréatif et patrimonial pour notre agglomération. Ces espaces sont inclus dans les objectifs de constitution d'un espace naturel métropolitain qui couvrira à terme environ to ००० ha dont 3000 conservent leur usage agricole.

Les zones agricoles et naturelles du sud de la métropole lilloise sont, tout particulièrement, l'objet de ces projections aménagistes depuis une quarantaine d'années [Amalric 2005; Franchomme et Kergomard 2006; Estienne op. cit.]. Il en résulte une juxtaposition de valeurs relatives à un foncier agricole tout à la fois support de production, gisement de la construction, infrastructures naturelles et répondant aux besoins récréatifs de la population citadine. Cette juxtaposition a eu comme corollaire de promouvoir la multifonctionnalité de l'espace et d'exacerber, au moins pour un temps, les conflits d'usages [Poulot 20I3]. Au regard du diagnostic du Scot laissant une place considérable aux circuits courts et à la fonction alimentaire de l'agriculture ${ }^{37}$, il semble que le postulat post-matérialiste soit plus présent que dans les documents précédents en promouvant une responsabilisation des modes de consommation individuels au profit d'une offre alimentaire de proximité.

\section{Des perspectives agricoles «nouvelles»}

Lévolution des modes de pensée aménagistes peut aussi se traduire par l'intégration de la Métropole européenne de Lille (MEL) au sein de réseaux nationaux et européens - comme Terres en villes, la plateforme Purple (Peri Urban Regions Platform Europe) - et l'organisation récente d'une concertation grand public sur la politique agricole et alimentaire de la Métropole (20I6). Cette mutation est légitimée par l'évolution du cadre de pratiques des activités agricoles. Les intérêts et les représentations des acteurs de l'aménagement et de la sphère agricole de ce que doivent être les espaces agricoles peuvent se rejoindre comme l'attestent de récentes prises de parole (discours du président de la chambre d'agriculture aux États généraux de l'alimentation à Oignies en novembre 20I7). Cette convergence se lit également dans la réalité des trajectoires agricoles locales. Une large fraction des exploitants agricoles de la MEL a développé de véritables stratégies d'insertion de l'agriculture dans l'espace urbain. Un tiers des exploitations mobilise aujourd'hui les circuits courts dans le Sud lillois ${ }^{3}$, alors que ce secteur est

37. Dans la partie 3 du diagnostic (dédiée à l'agriculture), la page 77 est intitulée «des opportunités pour l'agriculture périurbaine» et fait notamment mention du Schéma régional d'agriculture et d'alimentation durables, dont les objectifs portent sur les enjeux alimentaires de l'agriculture locale; les ressources foncières, «l'agriculture périurbaine d'excellence, la qualité de l'espace rural et de pratiques agricoles respectueuses de l'environnement».

38. Extraction des données RGA des 26 communes du parc de la Deûle et adjacentes. 
structuré par les cultures légumières ${ }^{39}$ de plein champ sous contrats, la production des pommes de terre (II2 producteurs pour I86 exploitations) et les grandes cultures. Pour plus de la moitié d'entre elles (34 sur 62), ces circuits courts représentent plus de Io \% du chiffre d'affaires (recensements généraux agricoles 2000-20I0). Au sein de ce même espace, émergent des figures nouvelles de l'agriculture: cueillettes à Seclin et à Avelin, pensions équestres (9), vente directe et transformation de produits laitiers ou porcins, de fruits et légumes... L'innovation passe, en matière d'initiative privée, par l'implantation du second magasin de circuit-court du réseau O'Tera dans la commune d'Avelin et par la création - et il s'agit là d'une initiative publique - d'une zone horticole et maraîchère à Wavrin.

Ces mécanismes d'ancrage territorial de la production agricole s'inscrivent dans un contexte général, sociétal (attentes nouvelles à l'égard des espaces ouverts et agricoles) et législatif ${ }^{\circ}$. Ils sont très largement commandés par le contexte foncier précédemment décrit et surtout conditionnées par les dynamiques propres aux bassins de production locaux (difficultés des filières endivière et laitière), par la recomposition des territoires de firme (délocalisation des cultures sous contrat vers des espaces plus ruraux) et par les opportunités de mise en marché. C'est la recherche de solutions d'adaptation des entreprises agricoles aux nouvelles conditions d'exercice de l'activité (réformes successives de la Politique agricole commune, imbrication croissante des usages agricoles, récréatifs, résidentiels, difficultés d'accès à la ressource foncière...) qui conduit les agriculteurs à substituer, en partie du moins, aux logiques de bassin de production, une logique d'intégration à un bassin de consommation. Les enquêtes, réalisées en 2005 en périphérie sud-est de l'agglomération (Pévèle-Mélantois), soulignent par ailleurs combien les trajectoires de reterritorialisation agricole ${ }^{4 \mathrm{I}}$ ont pu être initiées indépendamment de toute sollicitation des aménageurs. La formulation d'une demande explicite des élus et des décideurs à l'égard des agriculteurs succède aux réflexions engagées dans le contexte de révision du schéma directeur (I992-2002). Les inflexions de trajectoires agricoles, elles, ont le plus souvent été engagées avant la disqualification des systèmes de production en place. L'effondrement du cours des endives au milieu des années I980, le retrait des contrats des grandes entreprises agroalimentaires comme Bonduelle ou Avril dans les années I990 ou encore le processus de mise aux normes des élevages laitiers représentent trois temps forts qui ont

39. 84 exploitations sur un total de i86.

40. La loi d'orientation agricole de I999, loi de programmation relative à la mise en œuvre du Grenelle de l'environnement de 2009 , loi d'avenir pour l'agriculture, l'alimentation et la forêt (2OI4-II7O).

4I. Au sein des quatorze communes enquêtées, les deux tiers des agriculteurs tirent profit de la proximité urbaine: $\mathrm{I} 6 \%$ par la seule mise en œuvre de systèmes de production spécialisés, $50 \%$ par l'offre de service aux particuliers. 
effectivement pu conduire des chefs d'exploitation à accélérer leur conversion (du binôme endive-élevage vers le maraîchage en circuit-court, par exemple) ou leur tertiarisation (redéfinition et articulation des ateliers de production à une offre de services) [Rouget 2008].

La dynamique de renouvellement des liens à la ville a directement contribué à la reconnaissance de l'agriculture urbaine ou périurbaine et à la prise en compte de ses impératifs (sécurisation foncière). En effet, cette agriculture est, du point de vue aménagiste, redevenue utile non seulement en tant que producteur de biens publics (structuration et publicisation de l'espace [Le Caro et Kergreis 2007], paysages...), mais aussi en tant qu'opérateur privé capable de répondre aux attentes citadines, apte à produire une valeur ajoutée au territoire. Les inflexions en termes de trajectoires agricoles, la reconnaissance de l'agriculture comme activité à part entière du territoire métropolitain ainsi que la convergence des intérêts urbains et agricoles autorisent la formulation d'un objectif de co-construction des territoires. Il s'agit, également, de «faire territoire», de faire métropole, «de s'afficher dans la carte, de prendre rang» [Béhar et al. 20II] dans un contexte national où l'appropriation par les EPCI des questions relatives aux espaces et aux activités agricoles passe aussi par une mise en concurrence entre collectivités avec les appels à projets du Programme national pour l'alimentation ${ }^{42}$.

\section{Vers un nouveau paradigme du foncier agricole?}

Le principe de co-construction des territoires implique, au-delà d'évolutions concomitantes des représentations du territoire et du processus de convergence des intérêts, une dynamique collective d'appropriation d'un projet de développement territorial différencié 43 [François et al. 2013]. L'idée de co-construction s'est, à Lille, diffusée parallèlement à l'émergence et à la formalisation de projets d'aménagement d'envergure, ceux du parc de la Deûle, dont les ambitions initiales remontent à la fin des années I960. Ce projet, par ses dimensions temporelles et spatiales, peut être considéré aujourd'hui comme un laboratoire d'expérimentation du partenariat ville-agriculture autour de la question foncière.

\footnotetext{
42. Voir le site Internet (<http://agriculture.gouv.fr/pna-les-47-laureats-de-lappel-projet-2oI6 $-2017>)$.

43. Différenciation dont les agents économiques doivent eux-mêmes tirer profit, en l'occurrence [les] agriculteurs, par un gain en légitimité des demandes de mise en protection des surfaces agricoles, par également une différenciation-identification des produits et ce conformément aux logiques propres au concept de «ressource territoriale».
} 


\section{Le parc de la Deûle, un terrain d'expérimentation, mosaïque d'actions foncières}

Le parc de la Deûle a fait l'objet de plusieurs publications scientifiques s'attachant à l'analyser sous ses différentes dimensions: paysagères [Estienne op. cit.], agricoles [Serrano 20I2] et environnementales [Franchomme 2008]. Situé entre Lille et Lens, ce parc a pour finalité de combler le déficit en espaces verts et de loisirs de la métropole et de maintenir l'activité agricole périurbaine en contenant la poussée urbaine. Si le projet a été lancé en Ig68, l'inauguration n'a eu lieu qu'en 2004, soit près de quatre décennies plus tard, remplies d'actions foncières que l'on peut décomposer en cinq grandes phases éclairant l'actuel projet.

La première phase s'organise autour d'actions sporadiques de maitrise foncière, effectuées par les services de l'État et l'agence de l'eau dans les années I970. Ainsi, IIo ha sont acquis à l'intérieur de la zone de DUP (déclaration d'utilité publique) en 1972 et I973, suivis par 58 ha à Wingles au titre de la réserve foncière de l'État entre I973 et 1975.

Puis, durant la décennie I980, le projet sommeille en raison de son impopularité auprès des élus locaux, qui l'estiment trop «technocratique», et des agriculteurs qui craignent les expropriations. 90 ha sont pourtant acquis par des actions foncières éparses et font l'objet de conventions de mise à disposition et de gestion communale. Ainsi, bien que l'État soit, à la fin des années I980, propriétaire d'environ 350 ha, le projet ne peut être mis en œuvre.

Au début des années I990, Pierre Mauroy, alors maire de Lille, relance le projet et l'intègre dans une stratégie métropolitaine de reconquête de l'image des territoires ${ }^{44}$ en s'appuyant sur les municipalités et les acteurs publics locaux. Néanmoins, compte tenu du coût initial très élevé et du morcellement des préemptions, les acteurs réfléchissent à un nouveau projet.

Lélaboration du schéma directeur de 1998 est source de fortes tensions entre les représentants de la profession agricole et l'agence de développement et d'urbanisme. Ces frictions, qui marquent la quatrième phase, sont notamment déclenchées par plusieurs acquisitions foncières, à l'amiable ou par expropriation, de terres agricoles en vue de la protection des champs captants et de la définition même du périmètre du parc. Dans l'optique d'associer le monde agricole au projet et de contenir les anciennes velléités, les premières démarches de contractualisation et de charte de bonnes pratiques entre la métropole et les agriculteurs sont engagées dès i999.

Dans un cinquième temps, Lille métropole communauté urbaine (LMCU) crée, en 2002, le service communautaire Espace naturel et voies

44. À l'instar du contrat d'agglomération de 1990 entre l'État, la Région Nord-Pas de Calais et la Communauté urbaine de Lille. Cette coordination s'est poursuivie par un élargissement des compétences et du périmètre d'action de la métropole. 
d'eau (ENVE) et le syndicat mixte Espace naturel Lille métropole (ENLM). La LMCU confie au premier l'identification, la maîtrise et l'aménagement des terrains Espace naturel métropolitain (ENM) et, au second l'entretien, l'animation, la promotion des sites et la mise en œuvre du partenariat ville-agriculture. Le syndicat est constitué des collectivités (40 communes) ou groupements de collectivités adhérentes présentant un projet labellisé Espace naturel métropolitain conforme à l'objectif «Métropole Verte». La multifonctionnalité du parc est clairement revendiquée comme l'illustrent sur 400 ha les aménagements thématisés : la nature retrouvée (reconversion de sites industriels), domestiquée (agricole) et rêvée (artistique). Les éléments existants du paysage agricole (silos, hangars, clôtures, barrières, fossés...) sont également repris par les paysagistes du projet pour qu'ils composent la nouvelle identité des lieux. L'idée est de maintenir une activité agricole le long des cheminements du parc et des axes routiers reliant la ville au parc. Les agriculteurs sont mis à contribution soit par leur implication directe, en 2004, dans un projet de land art (réalisation de visuels, mise à disposition de terres dans le cadre de conventions établies pour l'inauguration du parc), soit par leur participation à deux programmes, portés par l'ENLM, d'intégration paysagère et de promotion des activités de diversification agricole (réseau de fermes du parc de la Deûle).

Le projet désormais consolidé, mais largement réduit en superficie ${ }^{45}$, nous éclaire sur l'importance de l'inertie induite par le morcellement de la propriété foncière et la volatilité du périmètre dans les projets urbains. Cette inertie est d'autant plus importante que les interventions foncières publiques portent sur des propriétés privées aux usages relatifs à des enjeux économiques et sociaux d'envergure. Le projet est aujourd'hui entré dans une nouvelle phase, formalisée à partir d'études prospectives amorcées en 2006 dans le plan de développement des espaces naturels métropolitains (PDENM). Il s'agit, d'une part, de mener, dans le tissu urbain, des actions foncières «en dentelle» sur d'anciens sites industriels et, d'autre part, d'affilier ou de labéliser ENM les espaces agricoles jouxtant le périmètre actuel du parc ${ }^{46}$. Les quelque 300 ha de terres agricoles le long du canal de Seclin sont visés par cette labellisation à moyen terme du fait de leur proximité avec le parc Mosaïc à forte affluence (nature rêvée). Les I Ioo ha des communes de Wavrin, Santes et Sainghin-en-Weppes sont identifiés pour des actions à long terme au titre de la ressource en eau, du cadre paysager et de l'agriculture de proximité (nature domestiquée).

45. Le projet initial s'étend sur 2500 ha et I3 communes.

46. Voir la Stratégie foncière de l'espace naturel métropolitain, Phase 1 et 2, Lille métropole communauté urbaine (LMCU), 2006 et l'État sur les potentialités d'extension du Parc de la Deûle, Espace naturel Lille métropole, 20 Io. 


\section{Une reconfiguration de l'organisation de l'acteur métropolitain}

Jusqu'à récemment, les espaces naturels relevaient du syndicat mixte ENLM et du service communautaire ENVE de la direction «Espace naturel et urbain». Le traitement des questions agricoles ne faisait pas l'objet d'un service dédié (voir fig. 6). La thématique était plus spécifiquement portée par le service «Planification urbaine et urbanisme» et plus précisément par un ingénieur chargé d'assurer la coordination des référents désignés dans toutes les directions de la MEL ayant un lien avec l'agriculture (développement économique, stratégie foncière, protection de la ressource en eau, espaces naturels...).

Les services en charge des espaces naturels et des questions agricoles ont fortement évolué en 2015 , puis en 2016 avec la mise en œuvre de la loi Mapam. Ainsi, le service «Espaces naturels et voies d'eau» devient l'unité fonctionnelle «Aménagement de la trame verte et bleue». Bien qu'elle reste dans le pôle «Aménagement et habitat», elle est rattachée à la direction «Urbanisme aménagement et ville» et en charge de la mise en œuvre du PDENM et du plan bleu. Elle a donc compétence sur la planification et la conduite des projets.

Le syndicat mixte, établissement territorial local, est dissous depuis le I5 avril 20I6. Ses agents sont intégrés au nouveau service «Espaces naturels métropolitains » et une mission «Agriculture et dynamiques territoriales » est créée au sein de la nouvelle direction «Nature, agriculture et environnement» du pôle «développement territorial et social». La transformation du syndicat en service métropolitain étend les prérogatives de ce dernier à l'ensemble des communes de la Métropole européenne de Lille et probablement à celles, moins urbaines et plus agricoles, qui seront incorporées avec les possibles élargissements successifs du périmètre de la métropole lilloise à l'horizon 2020. Il conserve la gestion des I 300 ha labellisés ENM. La direction en charge du foncier est subdivisée en un service dédié à l'action foncière et en une unité fonctionnelle en charge de l'élaboration des stratégies d'acquisitions et d'anticipation en lien avec les autres directions.

Cette évolution consacre de facto un rapprochement entre agriculture et espaces naturels. Elle favorisera un changement plus profond de politiques, dans lesquelles les projets articulant agriculture, ville et espaces naturels seront susceptibles d'être facilités ${ }^{47}$. Quelques inquiétudes subsistent néanmoins en termes de réactivité dans une vaste organisation comme la MEL. De plus, pour des raisons de calendrier, les orientations stratégiques pour les espaces naturels, d'une part, et les espaces agricoles, d'autre part, sont actuellement déclinées en deux processus organisationnels parallèles. Cependant,

47. Entretiens avec les représentants des services «espaces naturels métropolitains » et «stratégies et actions foncières ». 
ces deux stratégies convergent $4^{48}$, au moins partiellement, avec des politiques agricoles orientées vers l'alimentation de proximité et de labélisation des espaces agricoles en tant qu'«espaces naturels métropolitains». Actuellement, la révision générale du PLU en un PLU intercommunal conforme aux lois ENE et Alur devrait d'ailleurs intégrer cette convergence sur le foncier agricole apparente dans le Scot.

\section{Conclusion}

Avec le renouvellement des processus d'intégration du foncier agricole dans les préoccupations urbaines, il est essentiellement question, dans l'exemple lillois, d'une double adaptation. Adaptation de la sphère agricole à une métropolisation passant d'une phase de consommation de la terre agricole pour l'expansion de la ville à une volonté de maintien de cette dernière au titre du
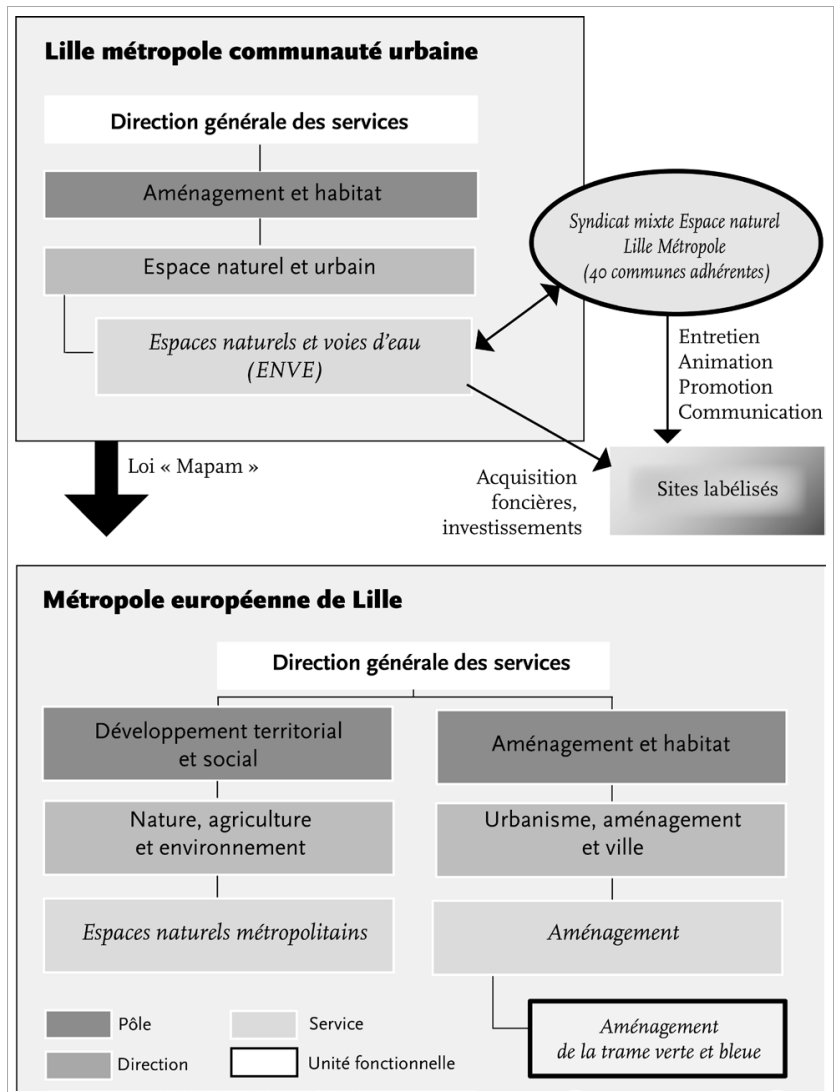

Figure 6.

Les évolutions récentes des services de la métropole lilloise en charge des espaces naturels.

48. Entretien avec le représentant du service «agricultures et dynamiques territoriales». 
cadre de vie. Adaptation des actions aménagistes avec une prise en considération des usages agricoles et de leurs tenants économiques suite à une période de tensions et de conflits avec les représentants de la sphère agricole durant les années 1990 et le début de la décennie suivante. La production alimentaire de proximité apparaît actuellement comme un axe de déploiement des politiques publiques dans la métropole lilloise dans la mesure où elle permet de concilier une agriculture productive et une multifonctionnalité des lieux (paysage, ressource...). Elle nécessite toutefois de reconsidérer les modes d'action et d'organisation. La récente juxtaposition au sein de la même direction des services «espaces naturels» et «agriculture» ainsi que la transformation d'un syndicat mixte de gestion des espaces naturels en service propre à la métropole s'expliquent avant tout par une logique gestionnaire. Néanmoins, qui dit rapprochement des services dit peut-être aussi rapprochement des thématiques par le biais d'enjeux transversaux comme les projets relatifs à l'agriculture de proximité (lotissement agricole à Wavrin, labélisation des terres agricoles jouxtant le parc de la Deûle, réseau de fermes partenaires...). Cette convergence n'offre pas pour autant à la dimension alimentaire des projets un rôle d'ensemblier consensuel des politiques publiques relatives à l'agriculture et aux espaces naturels. Les récents débats entre la majorité politique métropolitaine et les élus d'opposition autour de l'agriculture biologique et de la restauration collective attestent, en effet, que le foncier agricole reste le moyen et non l'objet du développement des espaces périurbains. Lélargissement du périmètre de la métropole lilloise vers des espaces moins urbanisés et plus agricoles pourrait concourir à une redéfinition des valeurs portées sur le foncier agricole dans un cadre intercommunal certes plus vaste sur le plan spatial, mais surtout avec des acteurs renouvelés considérant celui-ci non plus uniquement comme un support de projets urbains mais aussi comme une spécificité territoriale à valoriser.

Guillaume Schmitt géographe, maître de conférences à l'Université de Valenciennes, Cultures, Arts, littératures, Histoire, Imaginaires, Sociétés, Territoires, Environnement (EA 4343), ComUE Lille Nord de France, Valenciennes

Nicolas Rouget géographe, maître de conférences à l'Université de Valenciennes, Cultures, Arts, littératures, Histoire, Imaginaires, Sociétés, Territoires, Environnement (EA 4343), ComUE Lille Nord de France, Valenciennes

Magalie Franchomme géographe, maitre de conférences à l'Université de Lille, Territoires, Villes, Environnement et Société (EA 4477), Lille 


\section{Bibliographie}

Amalric, Marion, 2005,

Les zones humides, appropriations et représentations. L'exemple $d u$ Nord-Pas de Calais. Thèse de doctorat en géographie. Villeneuve-d'Ascq, Université Lille I.

Aubry, Christine, 20I3, «Les fonctions alimentaires de l'agriculture urbaine au Nord et au Sud. Diversité et convergence», Bulletin de l'Association de géographes français 90 (3): 303-3I7.

BARLes, Sabine, 2002, «Le métabolisme urbain et la question écologique», Les Annales de la recherche urbaine 92: I43-150.

\section{BÉHAR, Daniel, Philippe Estèbe et} Martin VANIER, 2OII, «Pôles métropolitains: du "faire territoire" au "faire politique", ou la nouvelle bataille de l'interterritorialité», Métropolitiques, I8 mai 20II (<www.metropolitiques.eu/ Poles-metropolitains-du-faire.html >).

Bousquet, Aurélie et al., 2013, «Les résolutions des bases de données "occupation du sol" et la mesure du changement. Articuler l'espace, le temps et le thème», L'Espace géographique 42 (I) : 6I-76.

Boutefeu, Emmanuel, 2005, La demande sociale de nature en ville. Enquête auprès des habitants de l'agglomération lyonnaise. Paris, Puca-Certu («Recherches» I54).

\section{Comber, Alexis, Peter Fischer} et Richard WADswORTH, 2004, «Integrating Land-cover data with Different Ontologies: Identifying Change from Inconsistency», International Journal of Geographical Information Science I8 (7) : 69I-708.

\section{Devillers, Rodolphe et Robert}

JeAnsoulin, 2005, «Qualité de l'information géographique: concepts généraux», in R. Devillers et R. Jeansoulin R. (dir.), Qualité de l'information géographique. Paris, Hermès Science («Traité IGAT. Géomatique»): 37-48.

Estienne, Isabelle, 2008, «La redécouverte de l'eau dans les stratégies d'aménagement à grande échelle de la métropole lilloise», Cahiers thématiques 6:250-263.

FrANCHомme, Magalie, 2008, Du cadastre napoléonien à la trame verte, le devenir des petites zones humides périurbaines en région Nord-Pas-de-Calais. Thèse de doctorat en géographie et aménagement. Villeneuve-d'Ascq, Université de Lille I.

Franchomme, Magalie et Claude KeRGOMARD, 2006, «Diversité régionale de la prise en compte des zones humides et de leurs dynamiques», Développement durable a territoires. «Dossier» 6 (<http://journals.openedition.org/ developpementdurable/I754>).

François, Hugues, Maud Hirczak et Nicolas SeniL, 20I3, «De la ressource à la trajectoire: quelles stratégies de développement territorial?», Géographie, économie, société I5 (3): 267-284.

\section{Geniaux, Ghislain et Claude} NAPOLÉONE, 2005, «Rente foncière et anticipations dans le périurbain», Économie \& prévision I68: 77-95. — 20II, «Évaluation des effets des zonages environnementaux sur la croissance urbaine et l'activité agricole», Économie Q Statistique 444-445: I8I-I99. 
GranelLe, Jean-Jacques, 2009 , Économie et urbanisme. Du foncier à l'immobilier 1950-2008. Paris, L'Harmattan (« L'esprit économique »).

GuIgou, Jean-Louis, I982, La rente foncière. Les théories et leur évolution depuis 1650. Paris, Economica.

HalberT, Ludovic, 20IO, L'avantage métropolitain. Paris, Presses universitaires de France («La ville en débat»).

Harvey, David, I989, «From Managerialism to Entrepreneurialism: the Transformation of Urban Governance in Late Capitalism », Geografiska Annaler 7I (I) : 3-I7.

Le Caro, Yvon et Sylvie Kergreis, 2007, «L'espace agricole comme espace public», in Y. Le Caro, P. Madeline et G. Pierre (dir.), Agriculteurs et territoires. Entre productivisme et exigences territoriales. Rennes, Presses universitaires de Rennes («Espace et Territoires»): 23I-250.

LE Roy, Étienne, I99I, «Introduction générale», in E. Le Bris, É. Le Roy et P. Matthieu (dir.), L'appropriation de la terre en Afrique noire. Manuel d'analyse, de décision et de gestion foncières. Paris, Karthala («Économie et Développement»): II-23.

LEROUX, Bertrand, 20IO, La planification spatiale aux prises avec le droit: le travail d'élaboration des schémas de cohérence territoriale.

Thèse de doctorat en aménagement de l'espace, urbanisme.

Champs-sur-Marne, Université Paris-Est.

LÉvy, Jacques, 20IO, «Le développement urbain durable entre consensus et controverse», L'information géographique 74 (3) : 39-50.
Margetic, Christine, Nicolas Rouget et Guillaume Sснмітт, 20I6, «Le foncier agricole à l'épreuve de la multifonctionnalité: desseins environnementaux et alimentaires dans les métropoles lilloise et nantaise», Norois 24I (4): 87-IO4.

\section{Martin, Samuel, Nathalie}

Bertrand et Nicole Rousier, 2006 , «Les documents d'urbanisme, un outil pour la régulation des conflits d'usage de l'espace agricole périurbain?», Géographie, économie, société 8 (3): 329-350.

PARIs, Didier, 1993, La mutation inachevée. Mutation économique et changement spatial dans le Nord-Pas de Calais. Paris, L'Harmattan («Géotextes»). — 2002, «Lille, de la métropole à la région urbaine», Mappemonde 66 (2): I-7.

Poulot, Monique, 20I3, «Du vert dans le périurbain. Les espaces ouverts, une hybridation de l'espace public (exemples franciliens)», EspacesTemps. net (<https://www.espacestemps.net/ articles/du-vert-dans-le-periurbain/>).

Rouget, Nicolas, 2008, Les dynamiques agricoles dans les espaces urbains et périurbains. Diversification et stratégies d'adaptation des agricultures. Les cas des périphéries sud-est de Lille et nord de Lens. Thèse de doctorat en géographie. Nanterre, Université de Paris-Nanterre. - 2013, «Trajectoires et stratégies agricoles dans les espaces urbains et périurbains. Entre spécialisation et conversion. L'exemple de la périphérie sud-est de l'agglomération lilloise», Bulletin de l'Association de géographes français 9o (3): 286-302.

Sснмітт, Guillaume, 2009, Valeurs et usages de l'espace: approches méthodologiques des dynamiques foncières dans la région Nord-Pas de Calais. Thèse de doctorat 
en géographie, aménagement de l'espace et urbanisme. Villeneuved'Ascq, Université de Lille I.

SERrano, José, 20I2, «L'insertion de l'agriculture dans les projets d'aménagement des élus urbains », Environnement urbain 6:32-49.
TorRe, André, 20I4, «L'agriculture de proximité face aux enjeux fonciers. Quelques réflexions à partir du cas francilien», Espaces et sociétés I58 (3): 3I-48.

\section{Résumé}

D'un foncier consumé à une réintégration des terres agricoles.

Le cas de la métropole lilloise

La succession des documents d'urbanisme, les politiques de planification, l'évolution même des principaux projets d'aménagement, les trajectoires agricoles laissent apparaître une inflexion des modes de penser le foncier et une reconsidération des valeurs et des fonctions attribuées aux terres agricoles des périphéries urbaines. De gisement de la construction celui-ci est aujourd'hui réhabilité dans sa fonction alimentaire. L'exemple de Lille est utilisé en raison de la précocité des réflexions sur le renouvellement des liens entre ville, nature et agriculture ainsi que de la richesse des expérimentations entreprises (parc de la Deûle notamment). Les reconfigurations en cours, y compris en matière d'organisation des services métropolitains, suggèrent une intégration croissante des politiques d'aménagement et de gestion des espaces ouverts.

Mots clefs: Lille, agriculture, alimentaire, foncier, nature, ville.

\section{Abstract \\ From consumed land to the reintegration of farmland. The case of metropolitan Lille.}

The succession of urban planning documents, planning policies, the evolution of major planning projects and agricultural trends point to a shift in how land is conceived and a rethinking of the values and roles attributed to farmland on urban peripheries. A shift has occurred from viewing such land as a pool for construction to seeking to restore its role as a food source. Lille was chosen because it is a precursor in rethinking the ties between the city, nature and farming, as well as for the wealth of experimentation that has occurred (Deûle Park, notably). The reconfigurations underway - including in terms of how metropolitan services are organized - suggest a growing inclusion of policies aimed at planning and the management of open areas.

Keywords: Lille, agriculture, food, land tenure, nature, city. 